\title{
Comparative Analysis of the Therapeutic Efficiency and Radiographic Measurement Between the Transforaminal Approach and Interlaminar Approach in Percutaneous Endoscopic Discectomy
}

\author{
Guodong YIN, Chong WANG, Shi-qin LIU \\ 923 ${ }^{\text {nd }}$ Hospital of People's Liberation Army, Department of Orthopedic Surgery, Nanning, Guangxi, P.R. China \\ Corresponding author: Guodong YIN ygd0810@126.com
}

\section{ABSTRACT}

AIM: To investigate and compare the therapeutic efficiency and radiographic measurement between the transforaminal approach and interlaminar approach in percutaneous endoscopic discectomy.

MATERIAL and METHODS: From January 2017 to January 2018, 86 patients suffering from single lumbar disc herniation were included in this retrospective analysis and divided into the percutaneous endoscopic transforaminal discectomy (PETD) group and percutaneous endoscopic interlaminar discectomy (PEID) group according to different surgical approaches. Data on age, gender, course of the disease, smoking status, and body mass index (BMI) were also obtained. Moreover, lumbar spine X-ray (anteriorposterior, lateral, and excessive flexion and extension), CT scan, and MRI were performed preoperatively for all patients. Another $\mathrm{MRI}$ of the lumbar spine was performed within the week after the operation. Clinical efficacy was calculated to assess the clinical effect of the therapy using the visual analogue scale (VAS) and Oswestry Disability Index (ODI) at 1 day before surgery, 3 months after surgery, 1 year after surgery, and the last follow-up visit.

RESULTS: All patients were followed up for above two year. 44 and 42 cases were enrolled, respectively, in the PETD and PEID groups. 62 males and 24 females were included in this study. The mean ages of the PETD and PEID groups were $34.58 \pm 6.70$ and $33.72 \pm 7.12$ years, respectively $(p=0.763)$. The symptoms showed evident improvement after surgery, but there were no significant differences regarding VAS scores, ODI scores, spinal canal occupation rate and lumbar lordosis except disc height and pfirrmann grade between the two groups ( $p>0.05)$. The revision surgery rates of the PETD and PEID groups at the last follow-up time were $2.3 \%$ and $4.8 \%$, respectively $(p=0.612)$. One patient in the PETD group underwent PEID revision surgery because of residual disk herniation. Re-operation were performed on 2 patients in the PEID group due to recurrencee.

CONCLUSION: Approaches of the PEID and PETD are pivotal to address lumbar disc herniation (LDH) disease. Selection of surgery approach depends on anatomical structure, physiological characteristic and operative skill of the surgeon.

KEYWORDS: Lumbar disc herniation, Percutaneous endoscopic transforaminal discectomy, Percutaneous endoscopic interlaminar discectomy, Treatment

\section{INTRODUCTION}

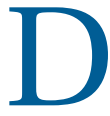

iscectomy is recommended when conservative treatments such as medication, injections, and physical therapy are not effective in treating patients suffering from lumbar disc herniation (LDH). The traditional operative procedure for LDH is open discectomy with or without lumbar fusion (2). However, numerous sequelae and slow recoveries may occur secondary to surgical trauma after open surgical intervention (6). 
Percutaneous endoscopic lumbar discectomy (PELD) is a new and full-endoscopic operative technique primarily used in the surgery for LDH. In contrast to the traditional open discectomy, the advantages of the PELD technique are as follows: smaller incision, preservation of the dorsal musculature and spine elements, less intraoperative hemorrhage, feasibility under local anesthesia, low morbidity rates, and rapid rehabilitation (24).

The procedures of PELD are usually performed using the transforaminal (18), or interlaminar (21) approach. Following the developing trends of endoscopic spine surgery, including surgical implements, optics design, and special operative approach, it has been more normalized and extensively used in clinical practice.

Although previous reports have compared the therapeutic outcomes between the two approaches in the treatment of LDH, the findings were not clear (12). Therefore, the aim of this study is to compare the therapeutic efficacy and the radiological results between the two approaches, which is critical in clinical decision-making on the basis of the patient's situation and the operator's surgical skills.

\section{MATERIAL and METHODS}

From January 2017 to January 2018, 86 patients suffering from single LDH were included in the retrospective analysis. 44 and 42 cases were enrolled, respectively, in the PETD and PEID groups on the basis of the corresponding surgical approaches. Data on age, gender, course of the disease, smoking status, and body mass index (BMI) were also obtained. Moreover, lumbar spine X-ray (anterior-posterior, lateral, and excessive flexion and extension), CT scan, and MRI were performed preoperatively for all patients. Another MRI of the lumbar spine was performed within the week after the operation. Clinical efficacy was calculated to assess the clinical effect of the therapy using the visual analogue scale (VAS) and Oswestry Disability Index (ODI) at 1 day before surgery, 3 months after surgery, 1 year after surgery, and the last follow-up visit. This study was approved by the medical ethics committee of the hospital. All operations were performed by the same associate chief physician who had experiences with over 300 cases of PELD.

The inclusion criteria were as follows: 1) unilateral radicular leg pain with or without lower back pain; 2) corresponding disk herniation shown on MRI; and 3) regular conservative treatment such as physical therapy, medication, and block therapy not effective in improving the symptoms in $>4$ weeks. The exclusion criteria were as follows: 1) revision surgery; 2) lumbar instability; 3) lumbar canal stenosis; 4) lumbar disk inflammation; 5) lumbar spinal tumor; and 6) multiple-level ( $\geq 2$ ) discectomy.

Indications for the PETD approach:

1) For lateral and extremely lateral disk herniation, PEID is unable to reach the intervertebral foramen area and its lateral surface because of the obstruction of the articular process, which has become a contraindication of PEID,
2) The diagnosis of LDH and lumbar spinal stenosis at the same lumbar level, except for severe central canal stenosis,

3) Disk herniation located on the $L 4 / 5$ level or above with narrow interlaminar window,

4) Revision surgery for patients with posterior open disk operation.

Indications for the PEID approach:

1) $L 5 / S 1$ segment with high iliac crest,

2) High-grade migrated nucleus pulposus,

3) Broad interlaminar window,

4) Axilla type of LDH: the nerve root is pushed outside by the protrusion. PEID can directly remove the protruded nuclear tissue through the axilla.

\section{Surgical Procedure}

PETD: Patients were put in a prone position and administered with basal anesthesia plus local anesthesia. An entry point was decided by the intersection of the horizontal line and the oblique caudal directional line tangent with the tip of the superior articular process (SAP). The approximate distances from the midpoint to the entry point were $6-8 \mathrm{~cm}$ for $L 3 / 4$ or L4/5 and 8-12 cm for LL5/S1. A 16G spinal needle was used in the piercing process, and the puncture trajectory was aimed at the SAP rather than the intervertebral foramen to avoid an iatrogenic injury in the ventral dural sac. Under monitored anesthesia care, $10 \mathrm{~mL}$ of $0.5 \%$ lidocaine was injected along the puncture route until the surface of the SAP. The cannula was pulled out when the stylet of the spinal needle was replaced by a guide wire. A stab skin incision was cut with a length of $7 \mathrm{~mm}$, focusing on the guide wire. Multilevel blunt guide rods were introduced along the guide wire step by step. The protective cannula was then inserted along the guide rod and leaned against the ventral surface of the SAP. Moreover, the guide rods were retrieved and replaced by a trephine to perform foraminoplasty. The trephine was pressed downward nearly horizontally and cranially to cut the ventral part of the SAP under fluoroscopic guidance. Afterward, the working cannula was finally placed in the incision (Figures 1, 2). When the protrusion was gradually removed, the traversing nerve root and dural sac were exposed with good mobility and good pulse, which indicated a complete decompression. Moreover, annuloplasty was performed to prevent relapse of herniation.

PEID: Patients were put in a prone position, and the PEID procedures were performed under basal and local anesthesia. A target point was determined at the outer area of the interlaminar window. A blunt dilator was inserted into the target point, and a working cannula was introduced outside of the ligamentum flavum (LF) under fluoroscopic guidance (Figures 3 , 4). The LF was opened using micropunches and forceps, and the working cannula was rotated while entering the lumbar spinal canal. The extruded nucleus pulposus should not be removed until the dural sac, compressed nerve root, and herniation location were confirmed and dissected to prevent neural injury. Moreover, the axillary or shoulder 


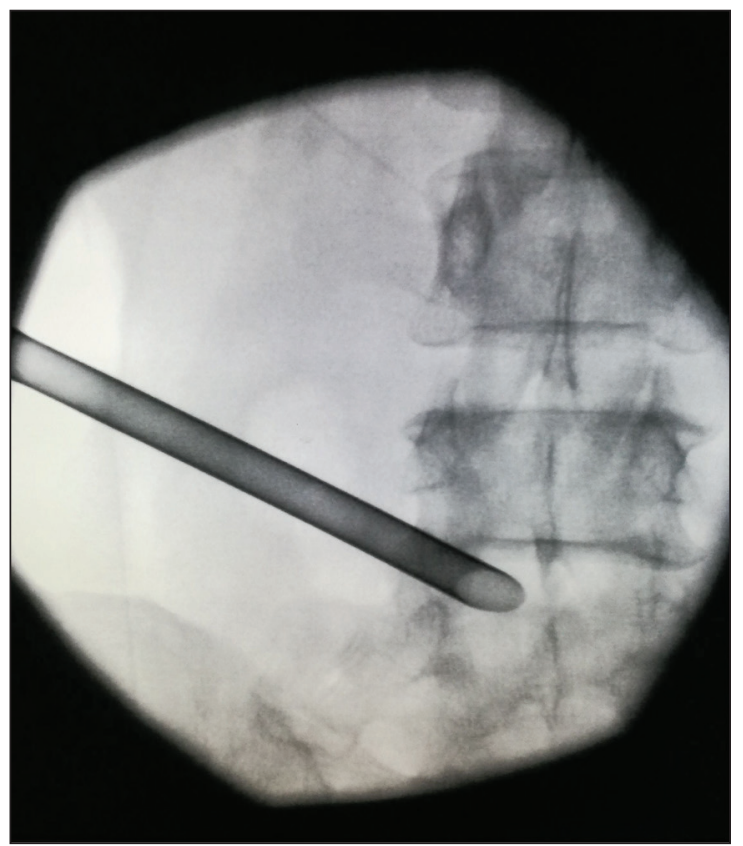

Figure 1: Posterior-anterior $(\mathrm{AP})$ radiography images showing placement of the working sheath (PETD).

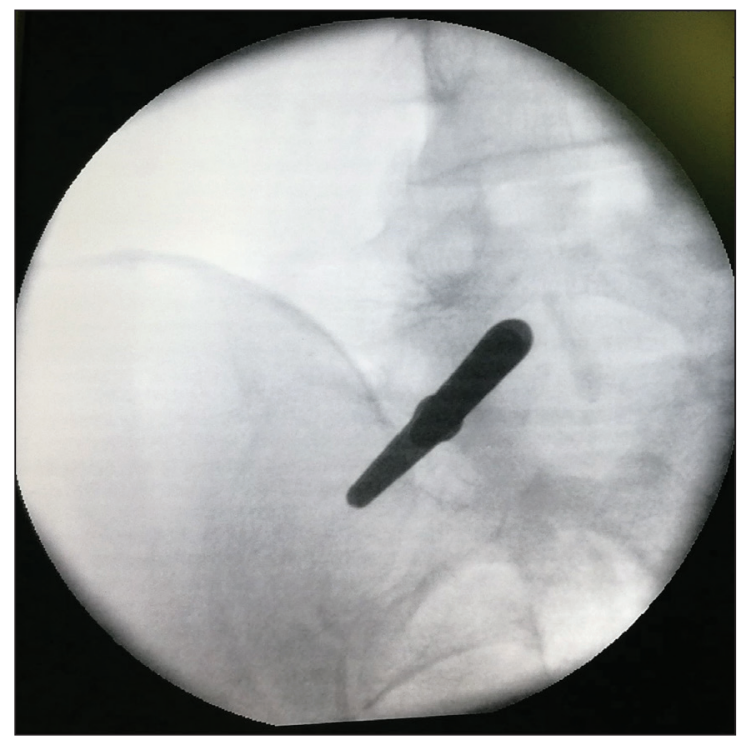

Figure 3: Posterior-anterior (AP) radiography images showing placement of the working sheath (PEID).

herniation and degenerative tissues were decompressed using grasping forceps to release the oppression and adhesion around the traversing nerve root.

\section{Statistical Analysis}

SPSS version 11.0 statistical package (SPSS Inc., Chicago, IL, USA) was used for statistical analysis. Significant difference was set at $p<0.05$. A Shapiro-Wilk test was conducted to assess the normality of the data. Parametric data were expressed as mean \pm standard deviation. Non-parametric

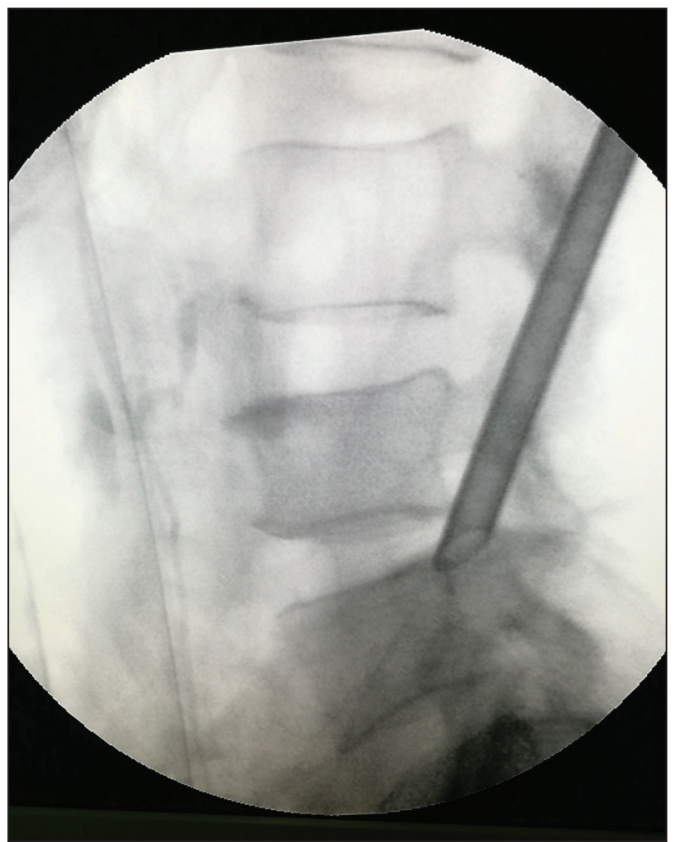

Figure 2: Lateral radiography images showing placement of the working sheath (PETD).

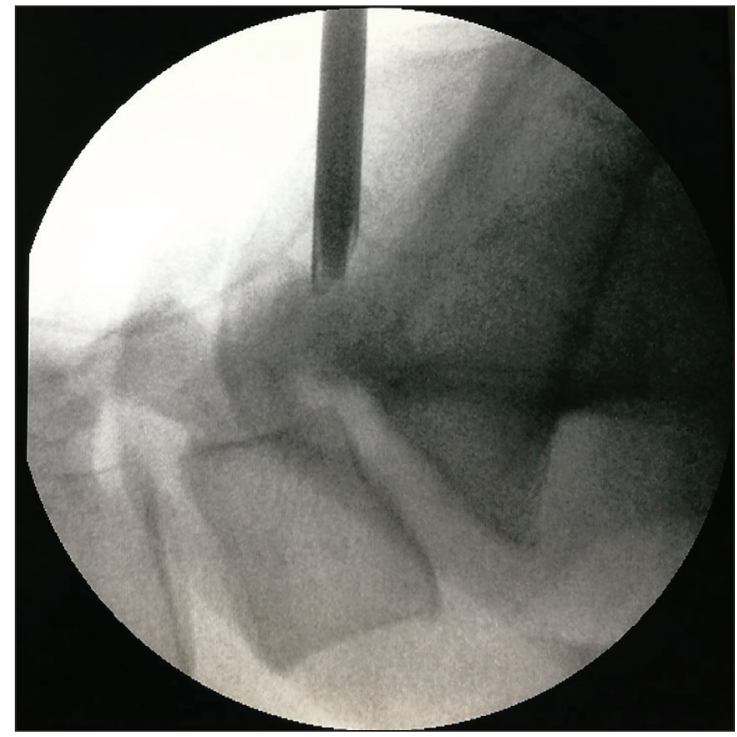

Figure 4: Lateral radiography images showing placement of the working sheath (PEID).

data were presented as medians (range). The categorical variables were presented as frequencies (\%) and processed using Fisher's exact test. Furthermore, the comparison of the parametric data was analyzed using one-way ANOVA or Student's t test. The Wilcoxon signed-rank test was used to analyze the non-parametric data.

\section{RESULTS}

No statistically significant differences were observed with 
regard to age, sex, course of the disease, smoking status, and BMI between the PETD and PEID groups. However, a significant difference was observed in the surgical level distributions between the two groups $(p<0.001)$. The PETD approach was more likely to be applied on the level of L3/4 or L4/5; the PEID, on the level of L5/S1. 62 males and 24 females were enrolled in the research. The mean ages of the PETD and PEID groups were $34.58 \pm 6.70$ and $33.72 \pm 7.12$ years, respectively $(p=0.763)$. Moreover, the durations of the symptoms in the PETD and PEID groups were $4.64 \pm 1.77$ and $4.85 \pm 1.69$ months, respectively $(p=0.559)$. The numbers of persons who smoke in the PETD and PEID groups were 6 $(13.6 \%)$ and $4(9.5 \%)$, respectively $(p=0.361)$. The BMls in the PETD and PEID groups were 21.71 and 20.90, respectively

Table I: Demographic Data

\begin{tabular}{lcc}
\hline Factors & Transforaminal & Interlaminar \\
\hline Age (years, mean \pm SD) & $34.58 \pm 6.70$ & $33.72 \pm 7.12$ \\
\hline Gender (female) & $10(22.7 \%)$ & $14(33.3 \%)$ \\
\hline Level & $4(9.1 \%)$ & $0(0 \%)$ \\
\hline L3/4 & $28(63.6 \%)$ & $6(14.3 \%)$ \\
\hline L4/5 & $12(27.3 \%)$ & $36(85.7 \%)$ \\
\hline L5/S1 & $4.64 \pm 1.77$ & $4.85 \pm 1.69$ \\
\hline $\begin{array}{l}\text { Duration of symptoms } \\
\text { (months) }\end{array}$ & $6(13.6 \%)$ & $4(9.5 \%)$ \\
\hline $\begin{array}{l}\text { Smoking } \\
\begin{array}{l}\text { Body mass index } \\
\text { (kg/m²) }\end{array}\end{array}$ & $21.71(17.6-32.0)$ & $20.90(16.5-30.9)$ \\
\hline
\end{tabular}

Normally distributed variables are presented as the means $\pm S D$; nonnormal continuous variables are presented as the medians (range); categorical variables are presented as frequencies (\%).
(Table I, $\mathrm{p}=0.208$ ). The operated levels were $\mathrm{L} 3 / \mathrm{L} 4$ in 4 cases (4.7\%), L4/L5 in 34 cases (39.5\%), and L5/S1 in 48 cases (55.8\%).

All procedures were performed successfully, and the 86 cases were analyzed with a mean follow-up of 26.4 months. The operation times of the PETD and PEID groups were 109.28 \pm 16.80 and $78.92 \pm 14.97 \mathrm{~min}$, respectively $(p<0.001)$. Moreover, the fluoroscopy times of the PETD and PEID groups were $13.10 \pm 3.85$ and $3.72 \pm 0.86$ times, respectively (Table II, $\mathrm{p}<0.001)$.

The postoperative and preoperative VAS scores of leg pain, as well as the ODI values, were displayed in Table III. The data (Figures 5-8) revealed that the postoperative VAS and ODI scores in both groups were significantly lower than those before operation at all follow-up time points, but no significant difference was observed between the two groups. MRI/CT examination indicated sufficient relief of the nerve root and the removal of the extruded nucleus pulposus (Figures 9-16).

No dysfunctional nerve root injury, dural tear, intestinal injury, iatrogenic segmental instability, or infection of the intervertebral disc was identified. The revision surgery rates of the PETD and PEID groups at the last follow-up time were $2.3 \%$ and $4.8 \%$, respectively $(p=0.612)$. One patient in the PETD group underwent PEID revision surgery because of residual disk herniation. Two cases in the PEID group presented with acute low leg pain at 4 and 4.5 months after surgery, which were diagnosed with recurrent herniation, and an open surgery was performed. Leg pain and lower back pain were relieved after performing pedicle screw fixation and posterior lumbar interbody fusion.

Compared with the preoperative results, the spinal canal occupation rate and disk height in both groups and the Pfirmann grade in the PEID group had a significant difference (Table IV, $\mathrm{p}<0.05$ ). In this study, it was found that the difference in the postoperative change of the lumbar lordosis in both

Table II: Comparisons of Operative Time and Fluoroscopy Time Between Transforaminal and Interlaminar Approach

\begin{tabular}{lcccc}
\hline & Transforaminal & Interlaminar & $\mathbf{t}$ & $\mathbf{p}$ \\
\hline Operative time & $109.28 \pm 16.80$ & $78.92 \pm 14.97$ & 5.353 & $<0.001$ \\
\hline Fluoroscopy time & $13.10 \pm 3.85$ & $3.72 \pm 0.86$ & 6.644 & $<0.001$ \\
\hline
\end{tabular}

p-value $<0.05$.

Table III: Comparisons of Clinical Data at Each Follow-Up Time Point Between Transforaminal and Interlaminar Approach

\begin{tabular}{lccccc}
\hline Group & Score & Preoperative & $\begin{array}{c}\text { 3 Months } \\
\text { postoperative }\end{array}$ & $\begin{array}{c}\text { 1 Year } \\
\text { postoperative }\end{array}$ & Final follow-up \\
\hline \multirow{2}{*}{ PETD } & ODI & $58.31 \pm 4.58$ & $26.02 \pm 2.44^{*}$ & $22.00 \pm 1.41^{*}$ & $20.00 \pm 1.55^{*}$ \\
\cline { 2 - 6 } & VAS & $8.07 \pm 0.76$ & $2.18 \pm 0.50^{*}$ & $1.52 \pm 0.59^{*}$ & $1.23 \pm 0.64^{*}$ \\
PEID & ODI & $57.00 \pm 3.70$ & $26.48 \pm 2.20^{*}$ & $21.81 \pm 1.89^{*}$ & $20.05 \pm 2.17^{*}$ \\
\cline { 2 - 6 } & VAS & $8.14 \pm 0.78$ & $2.48 \pm 0.74^{*}$ & $1.38 \pm 0.66^{*}$ & $1.24 \pm 0.43^{*}$ \\
\hline
\end{tabular}

Normally distributed variables are presented as the means $\pm S D$; ${ }^{*} p$-value $<0.05$ for pre-operation. 
Table IV: Comparisons of Radiographic Measurement Between the Two Groups

\begin{tabular}{lccccc}
\hline Index & \multicolumn{1}{c}{ Time } & Transforaminal $(\mathbf{t}, \mathbf{P})$ & Interlaminar $(\mathbf{t}, \mathbf{P})$ & $\mathbf{T}$ or $\mathbf{Z}$ & $\mathbf{p}$ \\
\hline \multirow{2}{*}{$\begin{array}{l}\text { Spinal canal } \\
\text { occupation rate }\end{array}$} & Pre-operation & $0.60 \pm 0.07$ & $0.63 \pm 0.14$ & -1.361 & 0.178 \\
\cline { 2 - 6 } Disc height & Post-operation & $0.20 \pm 0.06(41.514,<0.001)^{\star}$ & $0.21 \pm 0.09(16.333,<0.001)^{\star}$ & -0.337 & 0.737 \\
\hline \multirow{2}{*}{ Lumbar lordosis } & Pre-operation & $9.42 \pm 2.54$ & $8.45 \pm 1.77$ & 2.033 & 0.055 \\
\cline { 2 - 6 } & Post-operation & $8.70 \pm 1.51(3.550,<0.001)^{\star}$ & $7.31 \pm 1.52(9.482,<0.001)^{\star}$ & 3.887 & $\mathbf{0 . 0 0 0 * *}$ \\
\hline \multirow{2}{*}{ Pfirrmann grade } & Pre-operation & $39.05 \pm 11.50$ & $38.54 \pm 12.83$ & 0.195 & 0.846 \\
\cline { 2 - 6 } & Post-operation & $37.40 \pm 10.18(1.427,0.161)$ & $36.10 \pm 10.64(1.147,0.258)$ & 0.576 & 0.566 \\
\cline { 2 - 6 } & Pre-operation & $3.0(3.0,4.0)$ & $3.0(3.0,4.0)$ & -0.554 & 0.053 \\
\hline
\end{tabular}

Normally distributed variables are presented as the means $\pm S D$; non-normal continuous variables are presented as the medians ((interquartile range); ${ }^{*} p$-value $<0.05$ for pre-operation; ${ }^{* *} p$-value $<0.05$ compared between two groups.

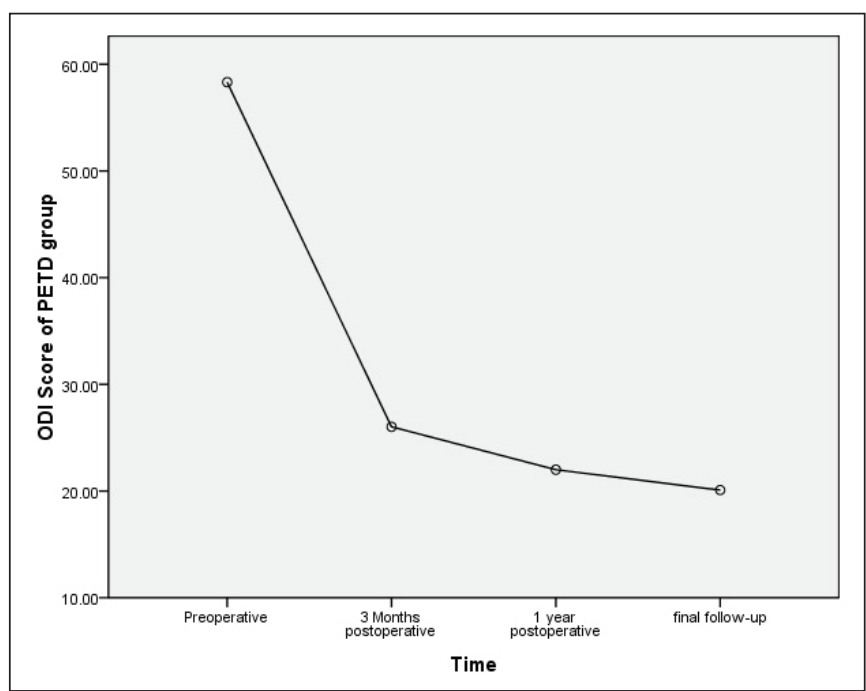

Figure 5: Curves of the mean scores on the ODI (PETD) before operation and at each time point postoperation.

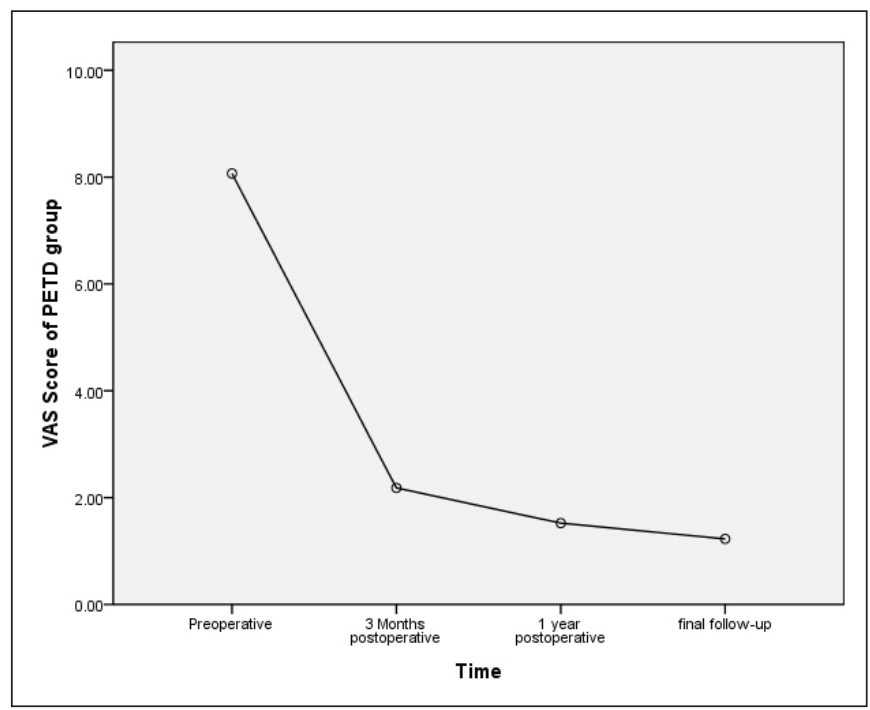

Figure 7: Graphs showing changes in the visual analog scale (PETD) before operation and at each time point postoperation.

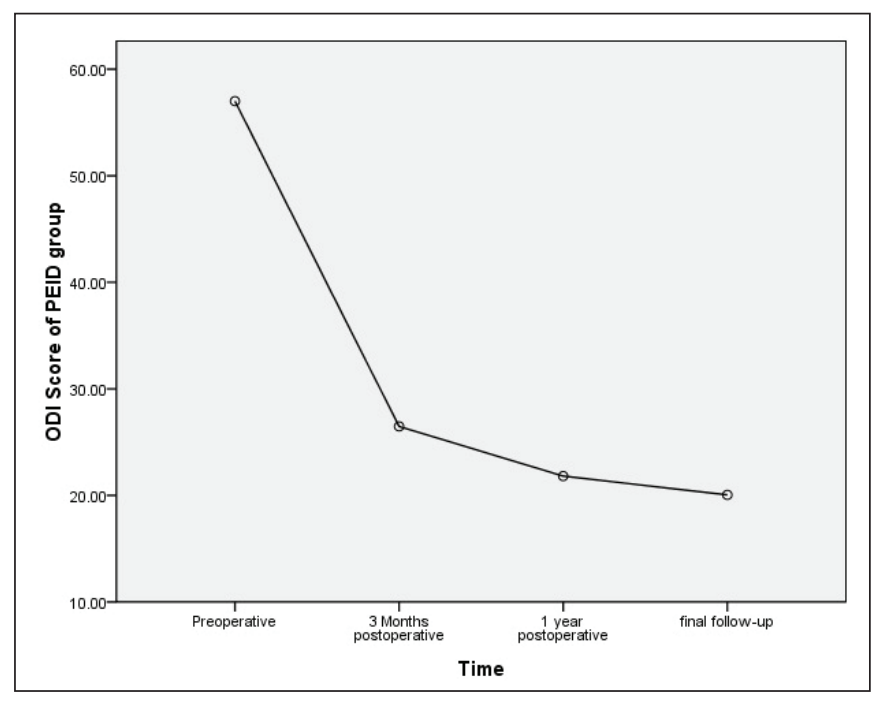

Figure 6: Curves of the mean scores on the ODI (PEID) before operation and at each time point postoperation.

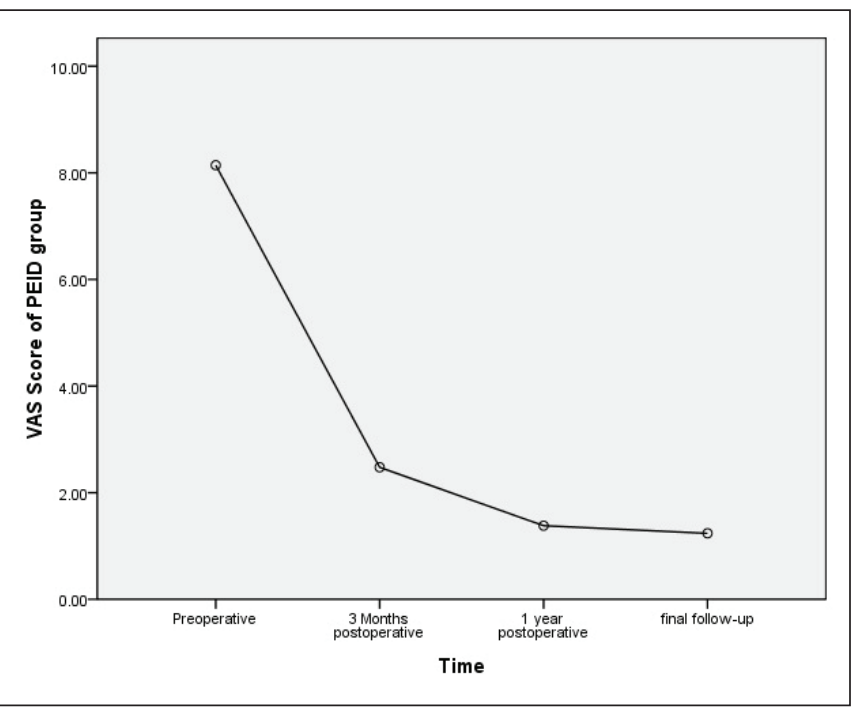

Figure 8: Graphs showing changes in the visual analog scale (PEID) before operation and at each time point postoperation. 


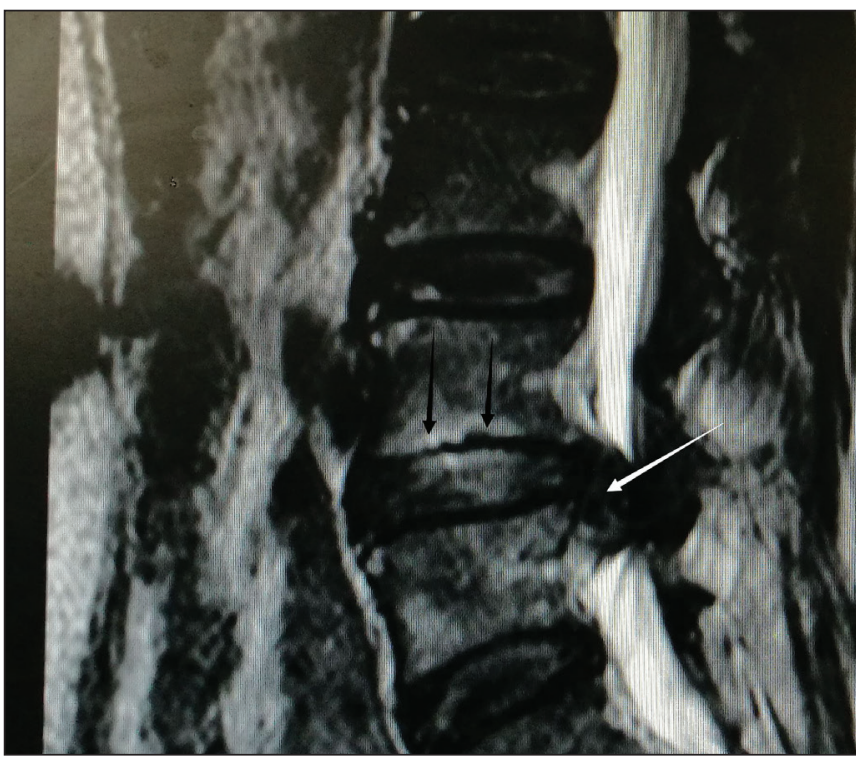

Figure 9: Examples of $L 4 / 5$ disc herniation. Sagittal T2 magnetic resonance imaging (MRI) showing large posteriorly extending L4/5 disc. The disc has marked T2 signal change and loss of fluid signal seen with disc dessication and fluid loss. The disc is extending directly posteriorly (solid white arrow). There is almost no narrowing of the vertical height of the disc space. There is early minor endplate Modic change in middle of L4/5 endplates (solid black arrow).

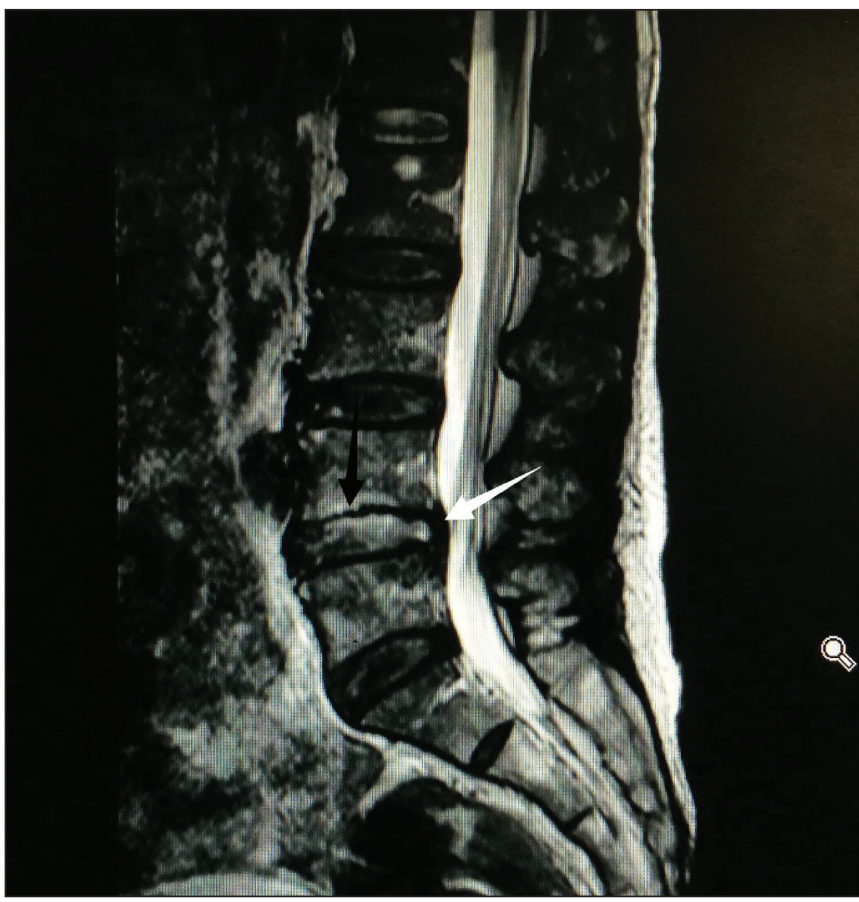

Figure 11: Postoperative Sagittal T2-weight MRI showing epidural space and nerve roots (solid white arrow) are clearly emerged compared to the preoperative MRI view. The posterior longitudinal ligament remains intact and the change of $L 4 / 5$ endplate (solid black arrow) state is not obvious.

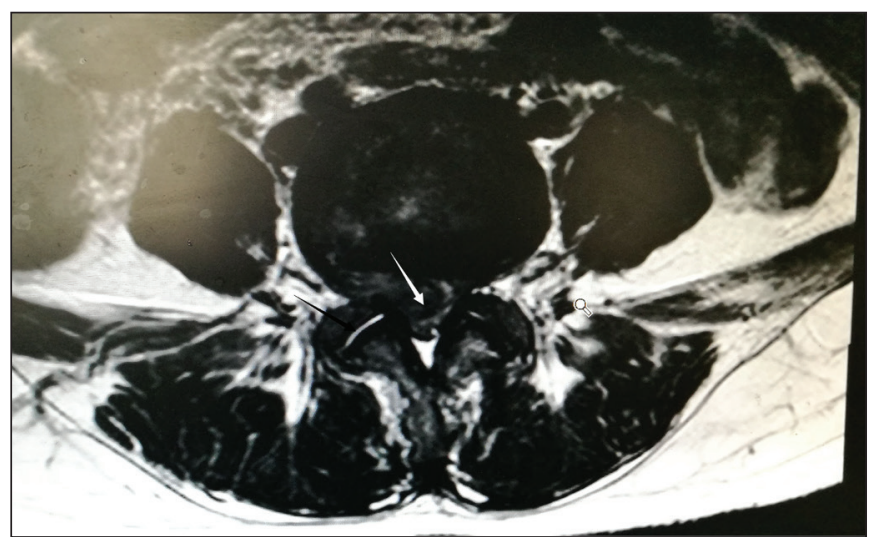

Figure 10: Examples of $L 4 / 5$ disc herniation. Axial T2 magnetic resonance imaging (MRI) showing large posterior herniation with T2 signal change in L4/5 disc. Larger herniation that is extruded into and occupied almost the epidural space (solid white arrow). There is minimal but distinct posterior ligamentum hypertrophy. There is high intensity fluid in both hypertrophy facet joints indicative of joint inflammation (solid black arrows).

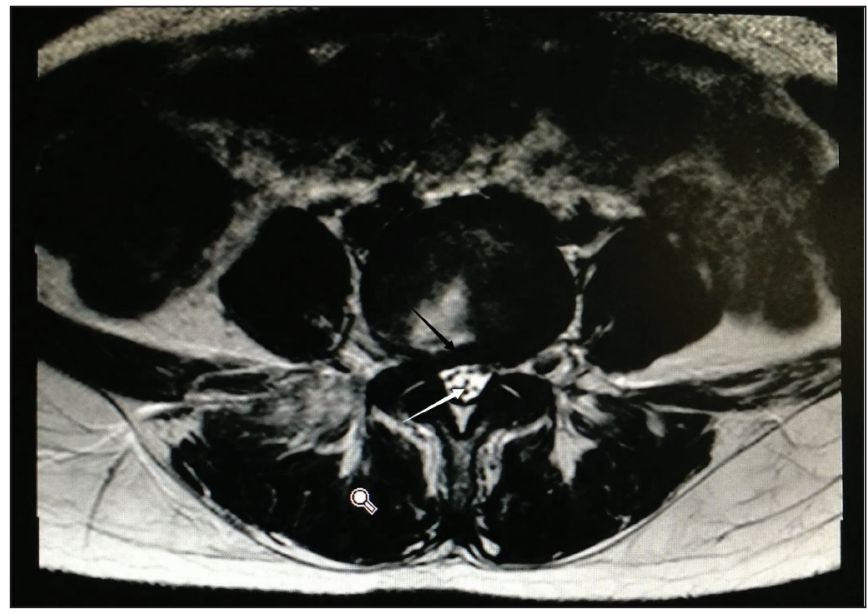

Figure 12: Postoperative axial MRI results indicating complete removal of the herniated disc material and the spinal canal volume enlarged. The cauda equina nerve (solid white arrow) is clearly visible in the spinal canal which was spaced with the decompressed portion of the L4/5 disc by the tough posterior longitudinal ligament and fibrous rings (solid black arrow).

groups and the Pfirrmann grade in the PETD group was not significant $(p>0.05)$. However, no significant difference was observed regarding the postoperative spinal canal occupation rate and lumbar lordosis between the two groups, except for the disk height and Pfirrmann grade between the two groups (Table IV).

\section{DISCUSSION}

After the introduction of the spinal endoscopy technique by Hijikata (10) and; Kambin and Sampson (14) in the 1980s, it has been widely known as a minimally invasive surgical alternative for spinal disk herniation. PETD has been applied for all kinds 


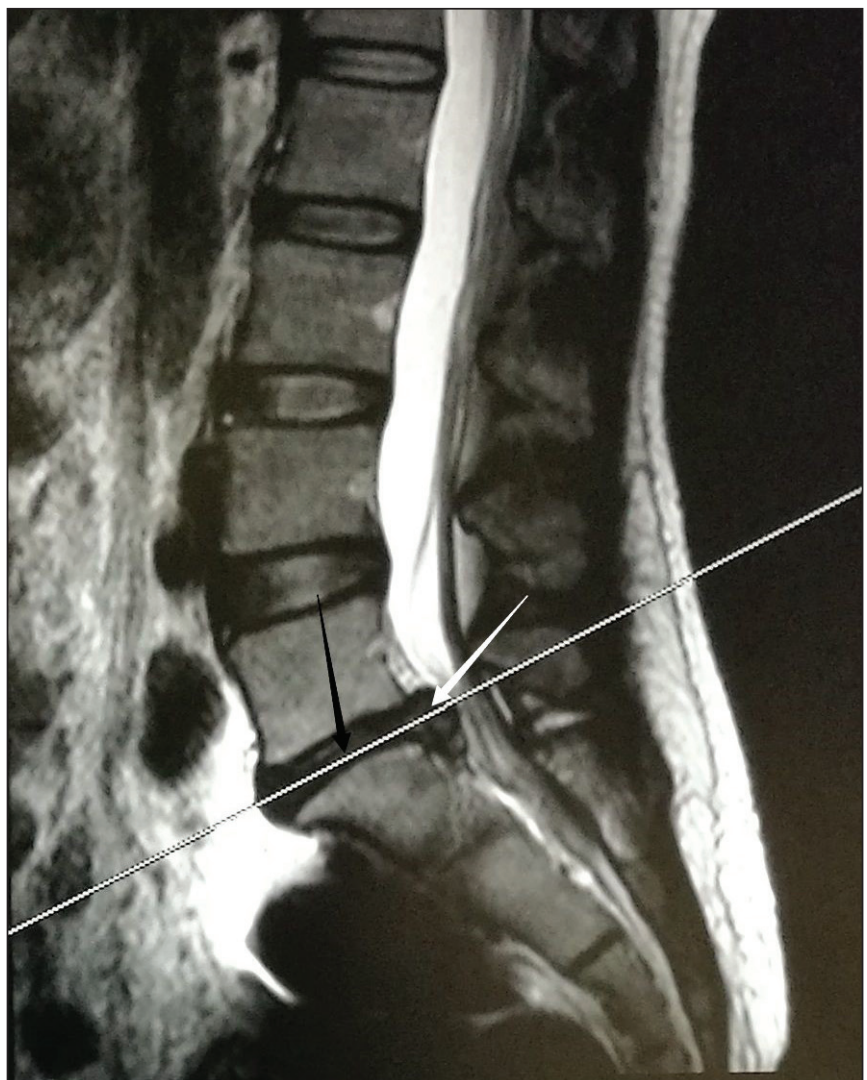

Figure 13: Examples of L5/S1 disc herniation. Sagittal T2 magnetic resonance imaging (MRI) showing moderate posteriorly extending L5/ $\mathrm{S} 1$. The disc has decreased T2 signal change (solid black arrow) and the herniation disc protrudes directly posteriorly (solid white arrow). There is moderate narrowing of the vertical height of L5/S1 disc and no Modic endplate change.

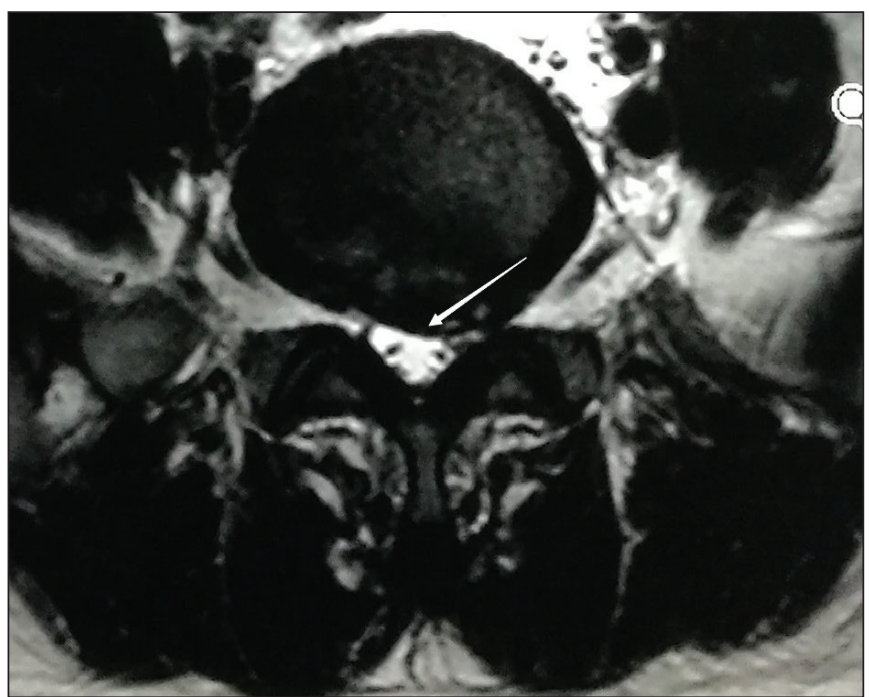

Figure 14: Examples of L5/S1 disc herniation. Axial T2 magnetic resonance imaging (MRI) showing posterior lateral extrusion (solid white arrow) with T2 signal change in L5/S1 disc. The left nerve root is squeezed against the posterior wall of the spinal canal by a herniated disc. The outline of the nerve could not be visible.

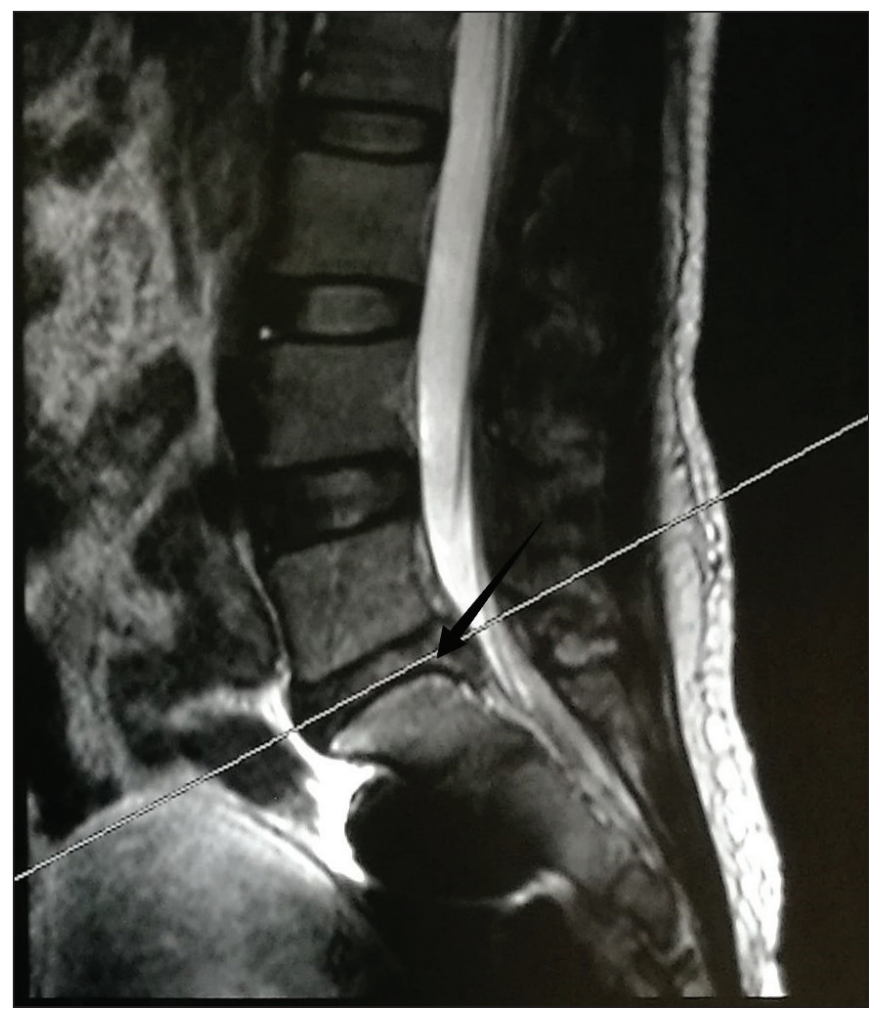

Figure 15: Postoperative Sagittal T2-weight MRI showing herniated disc decompression accomplishment (solid black arrow) compared to the preoperative MRI view, but the posterior edge of the disc seems to protrude into the spinal canal due to T2 signal change.

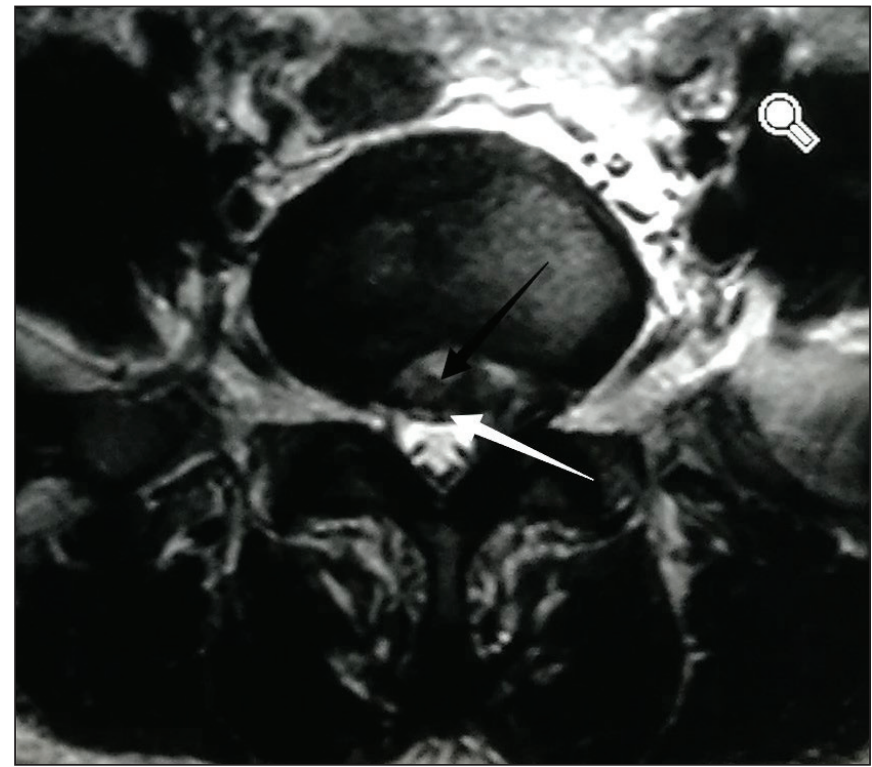

Figure 16: Postoperative axial MRI results indicating removal of the herniated disc material which is replaced by some mixing changes of high and moderate T2 signal (solid black arrow) . Nerve root decompression was well performed compared to the preoperative MRI view. But the "wall" formed by posterior longitudinal ligament and fibrous rings (solid white arrow) appears to be swelling (T2 signal change) and thin. 
of LDH at various lumbar segments, except for few special situations such as highly migrated nucleus pulposus and high iliac crest $(16,20)$. In addition, the above problems can be effectively resolved using the PEID approach $(15,27)$. The broad L5/S1 interlaminar space can provide free movement for the working sheath, allowing the surgical procedure to be performed as quickly as possible without excessive radiation exposure.

Some researchers have compared the clinical therapeutic efficiency and related influencing factors of the two methods, but the optimal option between the two methods is yet to be determined (11). Furthermore, this research was performed to assess the therapeutic effect and imaging changes of the two aforementioned approaches.

The results showed that it took approximately half an hour of extra operative time and nearly four times of radiation dose to complete the PETD surgery compared with the PEID surgery $(p<0.001)$. With regard to operative time and fluoroscopy time, Chen et al. analyzed six studies, including 399 patients, in a meta-analysis and came to a similar conclusion (8). This may be due to the following reasons (22):

1) Spine surgeons are more familiar with the PEID approach.

2) The PEID approach can provide a brief and rapid target location through a large L5/S1 interlaminar space under less fluoroscopy guidance.

3) The extruded or prolapsed disk tissues can also be dissected in a completely full vision.

As for the VAS and ODI scores, no significant difference between the two groups was found, but the scores in each group were significantly decreased at all postoperative time points, which suggested that both approaches were effective and comparable.

Previous researches indicated that a longer surgical time often meant a higher occurrence rate of complications, reoperations, and unplanned readmissions in the spinal operations $(3,9)$. Accordingly, to avoid the hazards of radiation exposure and complicated surgical procedures, more spinal surgeons preferred to choose an interlaminar approach, especially for the LDH of level L5/S1 $(4,25)$. Moreover, it was shown that the two groups had a significant difference in the surgical level distributions separately $(p<0.001)$. Previous reports did not find any significant difference between the PETD and PEID groups regarding incomplete decompression, recurrence, and incidence of overall complications $(8,11,19)$. In our research, we came to the same conclusion: the revision surgery rates in both groups had no significant differences $(p=0.612)$. Even so, the recurrence rate in the PEID group was $4.8 \%$, which was higher than that in the PETD group. We inferred that the possible reasons might be as follows:

(1) The PEID approach might have brought greater damage to the posterior longitudinal ligament and the fibrous ring in the process of disk decompression. However, PETD could achieve degenerative nucleus pulposus in the base of the herniated disc through a lateral disk incision, avoiding the destruction of the posterior structure.
(2) Less experience with PELD and the operations performed in the early developmental stage of PELD were believed to be unique risk factors for $\operatorname{LDH}$ recurrence $(23,26)$. As a result, the incidence of complications or revisions could be influenced by the difference in experience and proficiency $(1,13)$.

(3) Foraminoplasty can effectively enlarge the osseous lateral recess and prevent the potential recurrence of the root compression.

Moreover, the imaging results implied that the two approaches were validated methods for spinal canal decompression, but the differences in the degree of decompression were not significant between the two groups, which were also reflected in the similar decline of the ODI and VAS scores. Previous reports have revealed that the changes of lumbar lordosis are related to a variety of degenerative diseases of the lumbar spine, including spondylolysis and spondylolisthesis. The relationship between the lumbar disk degeneration and lumbar lordosis is not clear (5). On the basis of the research, no difference in the postoperative lumbar lordosis changes between the two approaches was observed. However, the significant difference in the changes of the disk height and Pfirrmann grade between the two groups might indicate a greater injury or degeneration of the disk in the PEID group $(7,12,17)$. The reason for this could be that the bony canal or lateral recess could be extended by performing foraminoplasty in the PETD group, which might sacrifice more disk tissues to meet the equivalent requirements for the decompression of the nerve root or dural sac in the PEID group.

\section{Main points:}

1. The comparative analysis of the therapeutic efficiency and radiographic measurement between the two approaches in PELD.

2. On the basis of the research, the patients in the PEID group were observed to have a higher recurrence rate than those in the PETD group, but no difference between the transforaminal and interlaminar approaches was observed.

3. The postoperative difference in the changes of the disk height and Pfirrmann grade between the two groups was significant, which indicated a greater injury or degeneration of the disk in the PEID group.

\section{Limitations:}

1. The research was retrospective, and the number of patients included in the study was not adequate, which might lead to bias. However, further randomized controlled trials with large sample sizes were necessary to validate the study.

2. One surgeon performed all of the operations in the research, and limitations in generalizability might still exist and lead to bias.

3. The disease severity and type of lumbar disc herniation were not considered, and a long-term follow-up study was required to identify the curative effect. 


\section{CONCLUSION}

Providing long-term optimum results of the endoscopic surgery therapy for LDH was not easy. In spite of the recurrence, residue, and disk degeneration like most intractable complications, PEID is pivotal in addressing LDH disease without having more extensive radiation exposure and is as effective as PETD. With respect to PETD, the learning curve was steeper, which set higher demands on the abilities of the performer, but the clinical outcomes had not been inferior (sometimes even superior) to PEID for LDH. Furthermore, the clinical decision of which operative approach to use is often influenced by the anatomical structure, physiological characteristic, and operative skill of the surgeon.

\section{ACKNOWLEDGEMENTS}

The paper was supported by the Natural Science Foundation of Guangxi Autonomous Region, project number 2017 GXNSFAA198364.

\section{REFERENCES}

1. Ahn SS, Kim SH, and Kim DW: Learning curve of percutaneous endoscopic lumbar discectomy based on the period (early vs. late) and technique (in-and-out vs. in-and-out-and-in): A retrospective comparative study. J Korean Neurosurg Soc 58: 539-546, 2015

2. Ahn Y: Endoscopic spine discectomy: Indications and outcomes. Int Orthop 43: 909-916, 2019

3. Ahn Y: Transforaminal percutaneous endoscopic lumbar discectomy: Technical tips to prevent complications. Expert Rev Med Devices 9:361-366, 2012

4. Ahn Y, Kim CH, Lee JH, Lee SH, Kim JS: Radiation exposure to the surgeon during percutaneous endoscopic lumbar discectomy: A prospective study. Spine (Phila Pa 1976) 38:617-625, 2013

5. Been E, Kalichman L: Lumbar lordosis. Spine J 14:87-97, 2014

6. Bogduk N, Aprill C, Derby R: Lumbar discogenic pain: Stateof-the-art review. Pain Med 14:813-836, 2013

7. Briseno MR, Phukan RD, Leonard DA, Herzog TL, Cho $\mathrm{CH}$, Schwab JH, Wood KB, Bono CM, Cha TD: The influence of adjacent level disc disease on discectomy outcomes. Eur Spine J 25:230-234, 2016

8. Chen J, Jing X, Li C, Jiang Y, Cheng S, Ma J: Percutaneous endoscopic lumbar discectomy for L5S1 lumbar disc herniation using a transforaminal approach versus an interlaminar approach: A systematic review and meta-analysis. World Neurosurg 116:412-420, 2018

9. Chen P, Hu Y, Li Z: Percutaneous endoscopic transforaminal discectomy precedes interlaminar discectomy in the efficacy and safety for lumbar disc herniation. Biosci Rep 39(2):BSR20181866, 2019

10. Hijikata S: Percutaneous nucleotomy. A new concept technique and 12 years' experience. Clin Orthop Relat Res 238:9-23, 1989

11. Huang Y, Yin J, Sun Z, Song S, Zhuang Y, Liu X, Du S, Rui Y: Percutaneous endoscopic lumbar discectomy for LDH via a transforaminal approach versus an interlaminar approach: A meta-analysis. Der Orthopäde 49:338-349, 2020
12. Jiang $X$, Zhou $X, X u N$ : Clinical effects of transforaminal and interlaminar percutaneous endoscopic discectomy for lumbar disc herniation: A retrospective study. Medicine (Baltimore) 97: e13417, 2018

13. Kafadar A, Kahraman S, Akboru M: Percutaneous endoscopic transforaminal lumbar discectomy: A critical appraisal. Minim Invasive Neurosurg 49:74-79, 2006

14. Kambin P, Sampson S: Posterolateral percutaneous suctionexcision of herniated lumbar intervertebral discs. Report of interim results. Clin Orthop Relat Res 207:37-43, 1986

15. Kim CH, Chung CK, Woo JW: Surgical outcome of percutaneous endoscopic interlaminar lumbar discectomy for highly migrated disk herniation. Clin Spine Surg 29:E259-E266, 2016

16. Kim HS, Yudoyono F, Paudel B, Kim KJ, Jang JS, Choi JH, Chung SK, Kim JH, Jang IT, Oh SH, Park JE, Lee S: Analysis of clinical results of three different routes of percutaneous endoscopic transforaminal lumbar discectomy for lumbar herniated disk. World Neurosurg 103:442-448, 2017

17. Lee SM, Lee GW: The impact of generalized joint laxity on the clinical and radiological outcomes of single-level posterior lumbar interbody fusion. Spine J 15:809-816, 2015

18. Mathews $\mathrm{HH}$ : Transforaminal endoscopic microdiscectomy. Neurosurg Clin N Am 7:59-63, 1996

19. Nie H, Zeng J, Song Y, Chen G, Wang X, Li Z, Jiang H, Kong Q: Percutaneous endoscopic lumbar discectomy for I5-S1 disc herniation via an interlaminar approach versus a transforaminal approach: A prospective randomized controlled study with 2-year follow up. Spine (Phila Pa 1976) 41 Suppl 19:B30-B37, 2016

20. Orita S, Inage K, Eguchi Y, Kubota G, Aoki Y, Nakamura J, Matsuura Y, Furuya T, Koda M, Ohtori S: Lumbar foraminal stenosis, the hidden stenosis including at L5/S1. Eur J Orthop Surg Traumatol 26:685-693, 2016

21. Ruetten S, Komp M, Godolias G: A New full-endoscopic technique for the interlaminar operation of lumbar disc herniations using 6-mm endoscopes: Prospective 2-year results of 331 patients. Minim Invasive Neurosurg 49:80-87, 2006

22. Wang B, Lu G, Patel AA, Ren P, Cheng I: An evaluation of the learning curve for a complex surgical technique: The full endoscopic interlaminar approach for lumbar disc herniations. Spine J 11:122-130, 2011

23. Wang H, Zhou Y, Li C, Liu J, Xiang L: Risk factors for failure of single-level percutaneous endoscopic lumbar discectomy. J Neurosurg Spine 23:320-325, 2015

24. Wang K, Hong X, Zhou BY, Bao JP, Xie XH, Wang F, Wu XT: Evaluation of transforaminal endoscopic lumbar discectomy in the treatment of lumbar disc herniation. Int Orthop 39:15991604, 2015

25. Xu B, Xu H, Destandau J, Ma X, He J, Xia Q, Ji N, Yang Q, Liu $\mathrm{Y}$, Jiang $\mathrm{H}$ : Anatomic investigation of lumbar transforaminal fenestration approach and its clinical application in far lateral disc herniation. Medicine (Baltimore) 96:e7542, 2017

26. Yao Y, Liu H, Zhang $H$, Wang $H$, Zhang $C$, Zhang Z, Wu J, Tang Y, Zhou Y: Risk factors for recurrent herniation after percutaneous endoscopic lumbar discectomy. World Neurosurg 100:1-6, 2017

27. Yeom KS, Choi YS: Full endoscopic contralateral transforaminal discectomy for distally migrated lumbar disc herniation. J Orthop Sci 16:263-269, 2011 